\title{
A Lab-based Multi-length Scale Approach To Characterize Lithium-ion Cathode Materials
}

\author{
S. R. Daemi ${ }^{\text {a }}$ and D.J.L. Brett ${ }^{\text {a }}$, P. R. Shearing ${ }^{\text {a }}$ \\ ${ }^{a}$ Department of Chemical Engineering, University College London, London, WC1E 6BT \\ UK
}

\begin{abstract}
X-ray computed tomography has emerged as a valuable tool to analyze battery materials. This paper explores the possibilities and limitations offered by a multi-length scale lab-based approach to study a lithium nickel manganese cobalt oxide electrode for lithium ion battery applications. The porosity, tortuosity and particle size distribution are extracted from micro- and nano-CT datasets and discussed in terms of the resolution available for the measurement. This work sets the basis for a range of studies to understand how different manufacturing routes affect the microstructure of the electrode and how this in turn affects battery performance.
\end{abstract}

\section{Introduction}

The global electrical capacity is approximately 20 terawatt hours and is mainly generated by the use of hydrocarbons such as natural gas and coal that produce a large amount of $\mathrm{CO}_{2}$ in the atmosphere upon combustion. To disrupt these damaging practices, novel technologies and ways of utilizing energy are being explored. A rise in the utilization of electric vehicles, renewable energy technologies and a further electrification of the grid are predicted to considerably increase the requirement for versatile energy storage technologies (1). Lithium-ion (Li-ion) batteries emerged as the leading energy storage devices for a range of applications towards the beginning of the 1990's and have since become the technology of choice for portable devices and consumer electronics due to the combination of their high power and energy densities (2). For the full implementation of Li-ion batteries in hybrid and electric vehicles, however, improvements are required in terms of energy density, cycle life, cost, safety and cycling rates (3).

The electrodes in many battery cells have a porous microstructure within which different phenomena occur. While a correct understanding of the morphology of these microstructures is fundamental in controlling battery performance, degradation and ageing, there is currently a lack of models that accurately describe the three-dimensional nature of these phenomena. Commonly used characterization techniques such as Scanning Electron Microscopes (SEM) (4) have been applied to analyze Li-ion batteries in a variety of situations. However, a lack of appreciation of three-dimensional features, as well as the failure to understand transport within porous networks, produces results and models that cannot accurately describe the electrochemical processes occurring in these complex 3D geometries. X-ray computed tomography (CT) has emerged as a promising technique to non-destructively analyze different battery materials as it allows imaging in a variety of working environments across a range of length-scales (5)-(7). 
This work presents a lab-based multi-length scale approach to characterize different aspects of a lithium nickel manganese cobalt oxide electrode in three dimensions using $\mathrm{X}$-ray CT. Uncalendared electrodes are examined in the micro- and nano-CT domains and various advantages and limitations related to these techniques are discussed in terms of sample preparation, imaging and modelling. This work intends to provide a toolbox for future studies to examine the effect of compression on battery electrodes.

\section{Experimental Methods}

\section{$\underline{\text { Sample Preparation }}$}

$\mathrm{LiNi}_{0.33} \mathrm{Mn}_{0.33} \mathrm{Co}_{0.33} \mathrm{O}_{2}$ (NMC) (Targray, Canada), conductive carbon black (Timical Super C65, Imerys Switzerland) and Polyvinylidene fluoride (PVDF) (Arkema France) were homogenized in a dual asymmetric centrifuge system (SpeedMixer DAC 150.1 FVZ-K, Hauschild Germany) with a 90:5:5 ratio, by mass. NMP (anhydrous, Sigma Aldrich) was used as a solvent. The slurry was then cast onto a $20 \mu \mathrm{m}$ thick aluminum sheet and spread with a doctor blade. The electrode sheet was then initially dried in an oven prior to further drying in-vacuo. The sheet was subdivided in a multitude of disks of different sizes according to the use.

The samples for micro-CT analysis were prepared by cutting a triangle from the electrode sample and attaching it to a pin with a two part epoxy (5 Minute Epoxy, ITW Devcon USA) and let cure for $20 \mathrm{~min}$. The electrode pillars for nano-CT are prepared on a laser micro-machining system (A Series Compact Laser Micromachining System, Oxford Lasers UK). A disk of $1 \mathrm{~mm}$ diameter was cut from a larger sheet with a biopsy bunch ( $1 \mathrm{~mm}$ biopsy punch, Miltex USA) and glued with the two part epoxy to a $1 \mathrm{~mm}$ dowel pin (HDP-1-12-A1, Accugroup UK) and let cure for $20 \mathrm{~min}$. This was then mounted into the lathe chuck in the laser micro-machining device. An in-depth description of the sample preparation technique can be found in (8).

\section{$\underline{\text { Tomography Scans }}$}

For the micro-CT analysis the samples were scanned in a lab-based micro-CT instrument (Xradia Versa 520, Carl Zeiss Inc.). For the nano-CT analysis the samples were scanned in a lab-based nano-CT instrument (Xradia Ultra 810, Carl Zeiss Inc.) The settings used for X-ray characterization are summarized in Table 1.

Table 1: The main scan settings used for X-ray CT characterisation

\begin{tabular}{|c|c|c|c|c|c|c|c|c|}
\hline Sample & $\begin{array}{l}\text { Scan } \\
\text { Type }\end{array}$ & $\begin{array}{l}\text { Magnification / } \\
\text { Imaging Mode }\end{array}$ & $\begin{array}{l}\text { Tube } \\
\text { Voltage } \\
(\mathrm{kV})\end{array}$ & Binning & $\begin{array}{l}\text { Pixel } \\
\text { Size } \\
(\mathbf{n m})\end{array}$ & $\begin{array}{l}\text { Number of } \\
\text { Projections }\end{array}$ & $\begin{array}{l}\text { Exposure } \\
\text { Time (s) }\end{array}$ & $\begin{array}{l}\text { Analysed } \\
\text { Volume } \\
\left(\mu^{3}\right)\end{array}$ \\
\hline NMC & $\begin{array}{l}\text { Micro } \\
-\mathrm{CT}\end{array}$ & $40 X$ & 100 & 2 & 370 & 2201 & 30 & $\begin{array}{l}331 \times 356 x \\
111\end{array}$ \\
\hline $\begin{array}{l}\text { NMC } \\
\text { Pillar }\end{array}$ & $\begin{array}{l}\text { Nano- } \\
\text { CT }\end{array}$ & $\begin{array}{l}\text { Large field of } \\
\text { view absorption }\end{array}$ & 35 & 2 & 126 & 1601 & 25 & $63 \times 64 \times 38$ \\
\hline
\end{tabular}

The micro-CT datasets were reconstructed using the FDK reconstruction algorithm in the Zeiss XMReconstructor software and the datasets were subsequently imported into 
Avizo. As the image quality in most cases allows for a clear definition of individual particles, only a 3D median filter was applied to remove sub-resolution noise. Segmentation was achieved with thresholding and magic wand tools. The contrast difference between the particles and the remaining carbon-binder-pore phase was used as a threshold for segmentation. Expansion and dilation operations were used to remove artefacts internal to particles. The largest internal volume was selected for morphological and transport parameter analysis and this value is quoted in Table 1. Morphological calculations such as particle size distributions and particles were calculated in Avizo Lite. The porosity and tortuosity factor were calculated with the Tau Factor MATLAB plugin (9). A representative volume element analysis was carried out to ensure the representativeness of the datasets. More information regarding this method is found in (10). The nano-CT datasets were also reconstructed using a parallel beam filtered back projection algorithm in Zeiss XMReconstructor and subsequently analyzed with the same analytical tools described for the micro-CT dataset.

\section{Results}
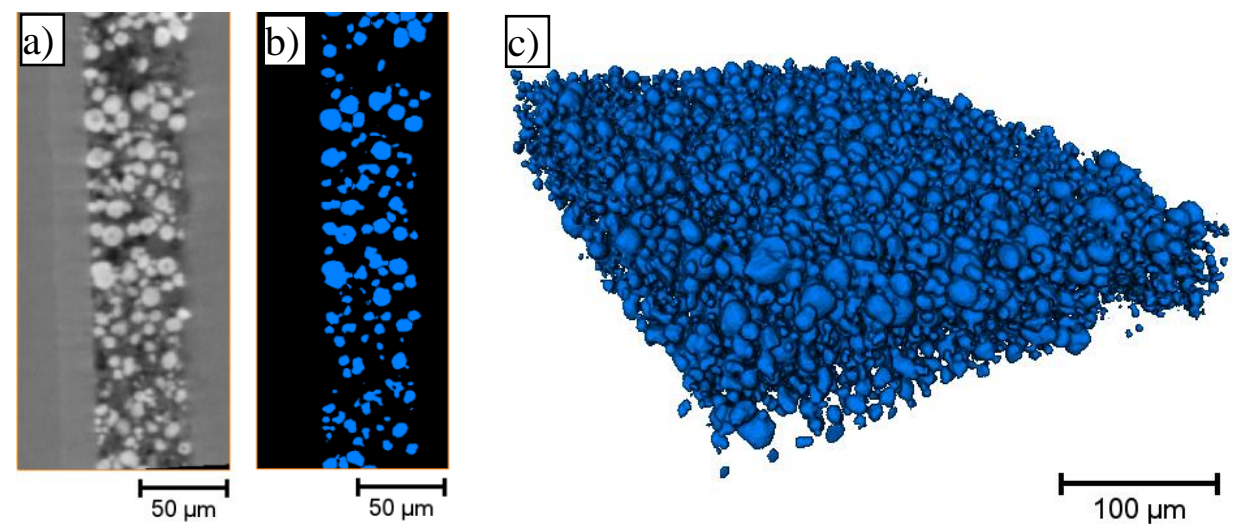

Figure 1: Virtual slices from the micro-CT dataset in a) original and b) segmented forms. The virtual slices are taken in the direction perpendicular to the current collector. c) Volume rendering of the dataset.

Figure 1 represents the micro-CT dataset in its original and segmented form as well as a $3 \mathrm{D}$ volume rendering for visualisation. The image represents a slice of the electrode throughout its thickness in respect to the current collector. The thickness of the electrode can be obtained by a 10 value average measured from the datasets and results in $54.9 \mu \mathrm{m}$. 


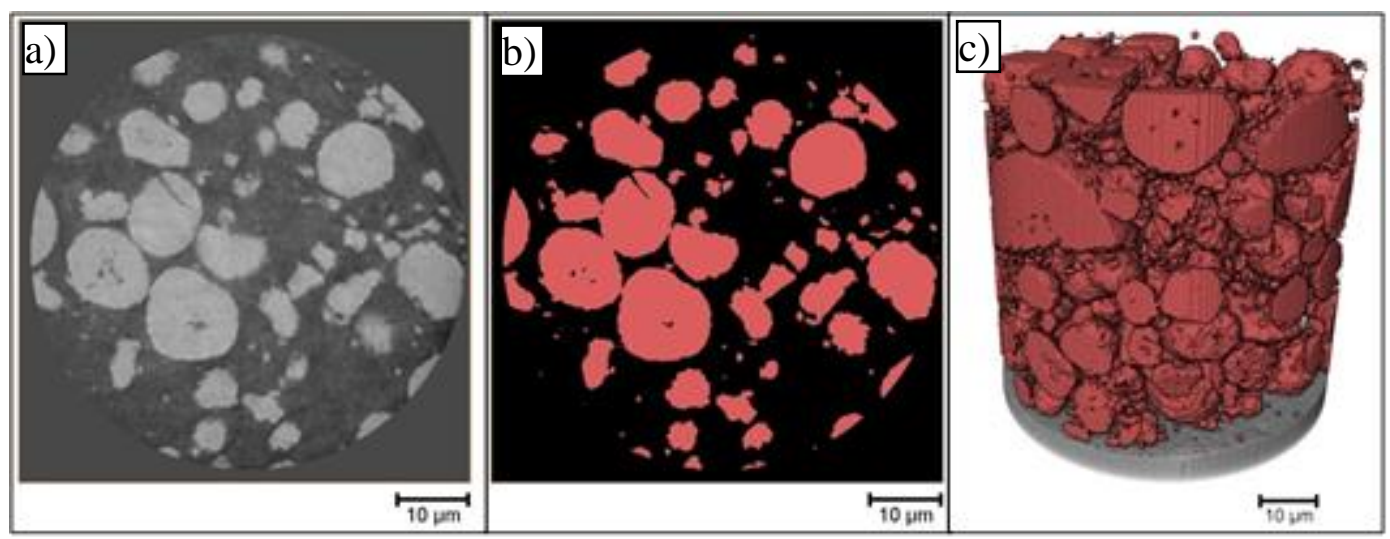

Figure 2: Virtual slices from the micro-CT dataset in a) original and b) segmented forms. Virtual slices taken in direction horizontal to the current collector. c) Volume rendering of dataset.

Figure 2 represents an xy slice through the micro-machined pillar along with the volume rendering. Features such as internal flaws and cracks are clearly visible in the nano-CT dataset, as well as a clear separation of the carbon/binder/pore phase from the active particle phase. The laser sample preparation technique offers an excellent method to prepare pillars for nano-CT analysis: the samples produced maintain the directionality and structure of an electrode as the current collector is still present, and this allows tailoring calculations to the relevant directions. Furthermore it is possible to tune the size of the pillar to maximize the amount of material within the field of view. A clear limitation of this dataset however is a lack of distinction between the carbon/binder and the pore phase.
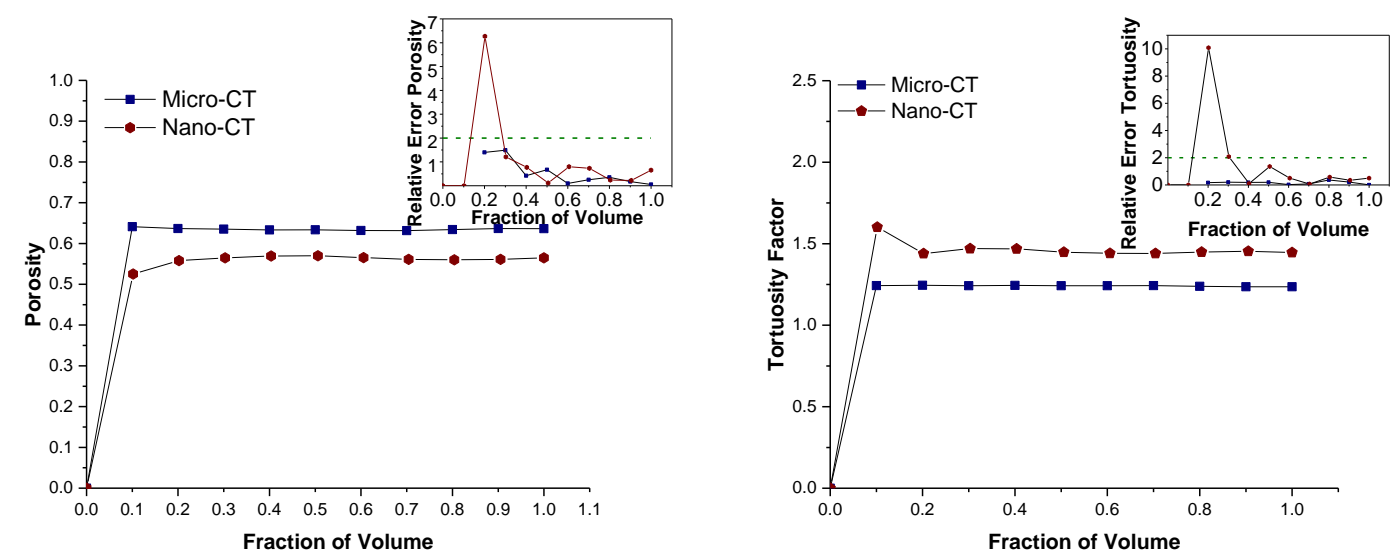

Figure 3: Porosity and Tortuosity factor for the micro- and nano-CT datasets calculated with the RVE approach.

Figure 3 represents the porosity and tortuosity calculated with a representative volume element (RVE) method. The RVE calculation is carried out to ensure that the dataset is sufficiently large to be deemed representative in respect to the property considered. As highlighted by both inset graphs, the volumes are sufficiently large to deem the calculated values as representative. The tortuosity is measured in the direction perpendicular to the current collector as this is relevant to the diffusion of Li-ions throughout the electrode. The porosity for the nano-CT scans is distinctly lower with values of $56 \%$ versus $64 \%$ for 
the micro-CT data. This can be explained by the fact that with the achievable resolution with the nano-CT instrument, the particle phase can be distinctly and accurately segmented from the $\mathrm{CBD}$, whereas at the micro-CT resolution, smaller particles and carbon-binder phase cannot always be discerned, leading to mis-segmentation of smaller fragments as the porous phase. Similar observations can be drawn for the tortuosity factor. A higher tortuosity factor of 1.46 for the nano-CT dataset is obtained compared to 1.24 for the micro-CT data. Again, this discrepancy in values can be associated to the difference in resolution between the two instruments used.

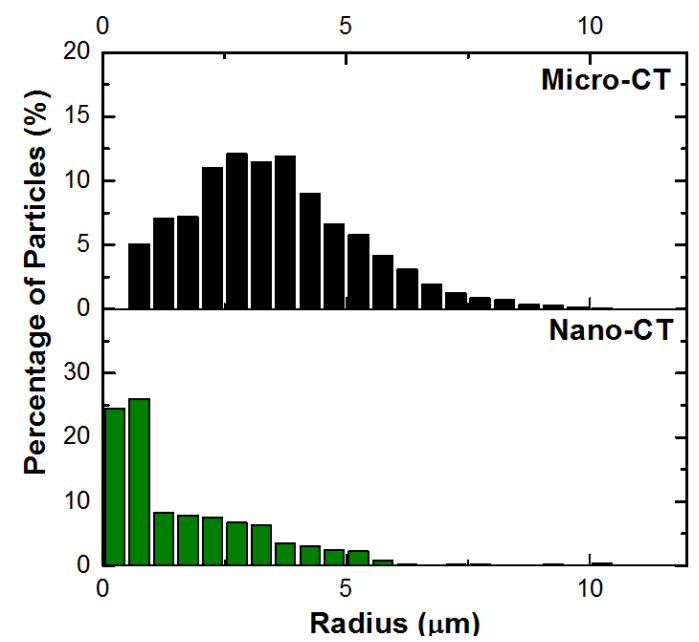

Figure 4: Particle size distribution for the micro- and nano-CT datasets obtained via the equivalent spherical radius method.

The effect of imaging resolution can also be observed in the particle size distribution in Figure 4. This is obtained through an equivalent spherical radius method. A higher amount of sub-micron particles are identifiable with the nano-CT scan as the pixel size is approximately 3 times smaller. Furthermore, there are a fewer particles at a larger radius for the nano-CT than the micro-CT dataset. This may indicate both a limitation of the micro-CT dataset to resolve the space between particles but also the lack of larger particles in the limited $65 \mu \mathrm{m}$ field of view of the nano-CT dataset.

\section{Conclusions}

This work has highlighted the potential in analyzing an NMC electrode from the micro- to the nano-CT scales using lab based equipment. Properties such as porosity, tortuosity and the particle size distribution were calculated and discussed as a function of imaging resolution. These results highlight the potential for characterization at both the electrode and particle level of these materials paving the way for analyzing electrodes manufactured following different routes and with different calendering techniques.

\section{Acknowledgments}

The authors acknowledge financial support from the EPSRC (EP/K005030/1, EP/N032888/1, EP/M009394/1) and UCL. 


\section{References}

1. BP, (2016).

2. J. B. Goodenough and Y. Kim, Chem. Mater., 22, 587-603 (2010).

3. J. Xu, S. Dou, H. Liu, and L. Dai, Nano Energy, 2, 439-442 (2013)

4. D. Chen, S. Indris, M. Schulz, B. Gamer, and R. Mönig, J. Power Sources, 196, 63826387 (2011)

5. D. S. Eastwood et al., Nucl. Instruments Methods Phys. Res. Sect. B Beam Interact. with Mater. Atoms, 324, 118-123 (2014)

6. S. J. Cooper et al., J. Power Sources, 247, 1033-1039 (2014)

7. P. R. Shearing et al., J. Electrochem. Soc., 159, A1023-A1027 (2012)

8. J. J. Bailey et al., J. Microsc., 0, 1-13 (2017)

9. S. J. Cooper, A. Bertei, P. R. Shearing, J. A. Kilner, and N. P. Brandon., SoftwareX (2016)

10. O. O. Taiwo et al., J. Microsc., 00, 1-13 (2016). 